\title{
Gust Response Characteristics of Helicopter Rotor Blade
}

\author{
Ma Hong-ru, Sun Wen-sheng, Mu Zhi-tao, Yan Guang-yao
}

(Department of Aeronautical Mechanism, Naval Aeronautical and Astronautical University Qingdao Branch, Qingdao 266041)

Keywords: helicopter; rotor blade; gust response

\begin{abstract}
The deformation of blades effected by gust would endanger the safety of wind turbine. Based on blades elastic deformation theory ,We established a model of blades rotation transient response ,studied the characteristics of blades transient response effected by gust, and confirmed the procedure of blades response to gust.
\end{abstract}

\section{Introduction}

Helicopter rotor always has to withstand gust wind when they are operating. High speed of gust would cause greater overload to helicopter rotor blades, which influences material load-carrying capability in negative way and would damage blades in advance. Consequently, during manufacture and service stages, the response characteristics of blades must be considered in order to ensure application security.

\section{Blades transient response model}

Blades response characteristics of gust prove to be a complex elastic deformation process. We applied the finite rotation beam theory to deal with problem of blades elastic deformation, established a kind of elaborate blade dynamics model ${ }^{[1]}$ using finite element method (FEM) in which We considered composite blades characteristics, key structure design parameters of blade (angle of cone, torsion angle, sweepback angle and etc), typical hub configuration characteristics and waving, pendulum vibration, torsion coupled motions and nonlinear deformation. When building the stain energy part, we introduced Danielson - Hodges generalized stain definition to decouple the three-dimensional nonlinear composite blades analysis into two-dimensional linear profile analysis and one-dimensional nonlinear analysis. While building the kinetic energy part, we introduced Hartenberg-Denarit augmented transformation matrix and raised a new recursion method on finding position vector of arbitrary point on a blade under the inertial coordinate system. Finally, according to the Hamilton principle, we established an nonlinear implicit dynamics equation in the form of generalized force.

\section{Model constructions}

\subsection{Strain energy item}

Considering the hypothesis of micro strain and using Danielson - Hodges definition, stress and strain force and moment of strain would respectively be:

$$
\boldsymbol{\kappa}=\boldsymbol{K}-\boldsymbol{k}, \quad \boldsymbol{\gamma}=\left[\boldsymbol{T}_{1}\right]\left[\begin{array}{c}
1+u^{\prime} \\
v^{\prime} \\
w^{\prime}
\end{array}\right]-\left[\begin{array}{l}
1 \\
0 \\
0
\end{array}\right]=\left(\begin{array}{l}
\overline{\boldsymbol{\varepsilon}}_{11} \\
\overline{\boldsymbol{\varepsilon}}_{12} \\
\boldsymbol{\varepsilon}_{13}
\end{array}\right)
$$

According to micro strain assumption, $\gamma_{m n} \cong \varepsilon_{m n}$, so the relationship of strain-displacement should be:

$$
\begin{aligned}
& \varepsilon_{12}=\left(\boldsymbol{G}_{12}-\boldsymbol{g}_{12}\right) / 2 \\
& \varepsilon_{13}=\left(\boldsymbol{G}_{13}-\boldsymbol{g}_{13}\right) / 2
\end{aligned}
$$


$\varepsilon_{11}=\left(\boldsymbol{G}_{11}-\boldsymbol{g}_{11}\right) / 2+2 \theta_{0}^{\prime}\left(\zeta \varepsilon_{12}-\eta \varepsilon_{13}\right)$

$G_{m n}$ and $g_{m n}$ are base vectors for deformed blades and undeformed blades, according to constitutive relation, the expression of strain energy variation should be:

$\delta U_{b}=\iiint_{V} \delta \varepsilon^{\mathrm{T}} \sigma d A d l$

$=\int_{0}^{l}\left[F_{x} \delta u_{e}^{\prime}+F_{y} 2 \delta \bar{\varepsilon}_{12}+F_{z} 2 \delta \bar{\varepsilon}_{13}+M_{x} \delta \phi^{\prime}+M_{y} \delta \kappa_{2}+M_{z} \delta \kappa_{3}\right] \mathrm{d} l$

Using the strain relationship listed above, section force and moment would be:

$\left(\begin{array}{c}F_{x} \\ F_{y} \\ F_{z} \\ M_{x} \\ M_{y} \\ M_{z}\end{array}\right)=\left[\begin{array}{llllll}T_{11} & T_{12} & T_{13} & T_{14} & T_{15} & T_{16} \\ T_{12} & T_{22} & T_{23} & T_{24} & T_{25} & T_{26} \\ T_{13} & T_{23} & T_{33} & T_{34} & T_{35} & T_{36} \\ T_{14} & T_{24} & T_{34} & T_{44} & T_{45} & T_{46} \\ T_{15} & T_{25} & T_{35} & T_{45} & T_{55} & T_{56} \\ T_{16} & T_{26} & T_{36} & T_{46} & T_{56} & T_{66}\end{array}\right]\left(\begin{array}{c}u_{e}^{\prime} \\ -\bar{\varepsilon}_{12} \\ 2 \bar{\varepsilon}_{13} \\ \phi^{\prime} \\ \kappa_{2} \\ \kappa_{3}\end{array}\right)=[\boldsymbol{T}]\left(\begin{array}{c}u_{e}^{\prime} \\ 2 \bar{\varepsilon}_{12} \\ \bar{\varepsilon}_{13} \\ \phi^{\prime} \\ \kappa_{2} \\ \kappa_{3}\end{array}\right)$

Matrix T refers to profile stiffness matrix and could be calculated by using Giavotto ${ }^{[2]}$.

By substituding strain-displacement relationship into the expression of elastic potential energy, we could separate every elastic generalized force caused by the elastic potential energy. Then partial derivation of every generalized force in every generalized coordinates is solved, and we could gain stiffness matrix caused by elastic potential energy.

\section{2 the aerodynamic force Item}

If on the inertial coordinates any blade variable axis point velocities are avaiable and rotor plane airflow is projected onto the same inertial coordinate system, we could calculate relative local airflow velocity of arbitrary point on blade variable axis and then transform it into deformed coordinate system to gain the velocity composite. According to Leishman - Beddoes two-dimensional unsteady and dynamic stall model, we could calculate the blade section flow velocity and angle of attack, and then calculate the section lift, resistance and pitch moment,

finally calculate virtual work of aerodynamic force and generalized force created by aerodynamic force.

Aerodynamic lift L, resistance D and moment M, respectively are:

$L=\frac{1}{2} \rho_{a} V_{P_{0}}^{2} c C_{N}$

$D=\frac{1}{2} \rho_{a} V_{P_{0}}^{2} c C_{C}$

$M=\frac{1}{2} \rho_{a} V_{P_{0}}^{2} c^{2} C_{M}$

Aerodynamic force in the hub rotating coordinates could be expressed as:

$\boldsymbol{F}_{A}=\left[\begin{array}{lll}F_{x} & F_{y} & F_{z}\end{array}\right]=\left[\begin{array}{lll}0 & -D & L\end{array}\right] \boldsymbol{T}_{1} \boldsymbol{T}_{s} \cdots \boldsymbol{T}_{f} \boldsymbol{T}_{r}$

Virtual work variation done by Air power $\mathrm{F}_{\mathrm{A}}$ and moment $\mathrm{M}_{\mathrm{A}}$ could be expressed as:

$\delta W_{A}=\int_{l}\left(\boldsymbol{F}_{A} \cdot \delta \vec{r}_{P}^{\mathrm{T}}+\boldsymbol{M} \cdot \delta \alpha\right) \mathrm{d} x=\sum_{i=1}^{18} Q_{i}^{A} \delta q_{i}$

\section{3 blade dynamical equation}

By using Hamilton principle, rotor system motion equation would be:

$\int_{t_{1}}^{t_{2}}\left[\delta\left(U_{b}-T_{b}\right)-\delta W_{A}\right] \mathrm{d} t=0$

Introducing Chopra finite element method (FEM) to scatter the 15 degrees of freedom of beam element and to gather kinetic energy strain energy and aerodynamic virtual work, we could gain blade implicit nonlinear dynamics differential equation based on generalized force, which could be expressed as follow: 
$Q_{i}^{E}(q)+Q_{i}^{T}(q, \dot{q}, \ddot{q}, t)+Q_{i}^{A}(q, \dot{q}, t)=0$

$\left(i=1,2, \ldots, n_{b}\right)$

\section{4 gravity item}

Blade gravity couldn't be ignored, while we deal with problems like blade raising or dropping. External work caused by blade gravity would be:

$$
\begin{aligned}
& \delta W_{G}=\iint_{l} \int_{A} \rho_{b} \boldsymbol{g} \cdot \delta \boldsymbol{R}_{n} \mathrm{~d} A \mathrm{~d} l \\
& \quad \text { generalized force generated by blade gravity would be: } \\
& Q_{i}^{G}=\int_{l} \iint_{A} \rho_{b} \boldsymbol{g} \cdot \frac{\partial \boldsymbol{R}_{n}}{\partial q_{i}} \mathrm{~d} A \mathrm{~d} l
\end{aligned}
$$

\section{Calculation results and analysis}

Based on the above theoretical analysis, a certain type of blade response characteristics effected by gust could be calculated . Figure 1 shows changes of blade tip waving quantity during starting process. In this process, because of low rotate speed and small centrifugal force, the blade tip expresses wide waving movement effected by gust. with the increase of rotational speed, centrifugal force also increases, under the effect of centrifugal force, waving range decreases gradually and tends to a stable range.

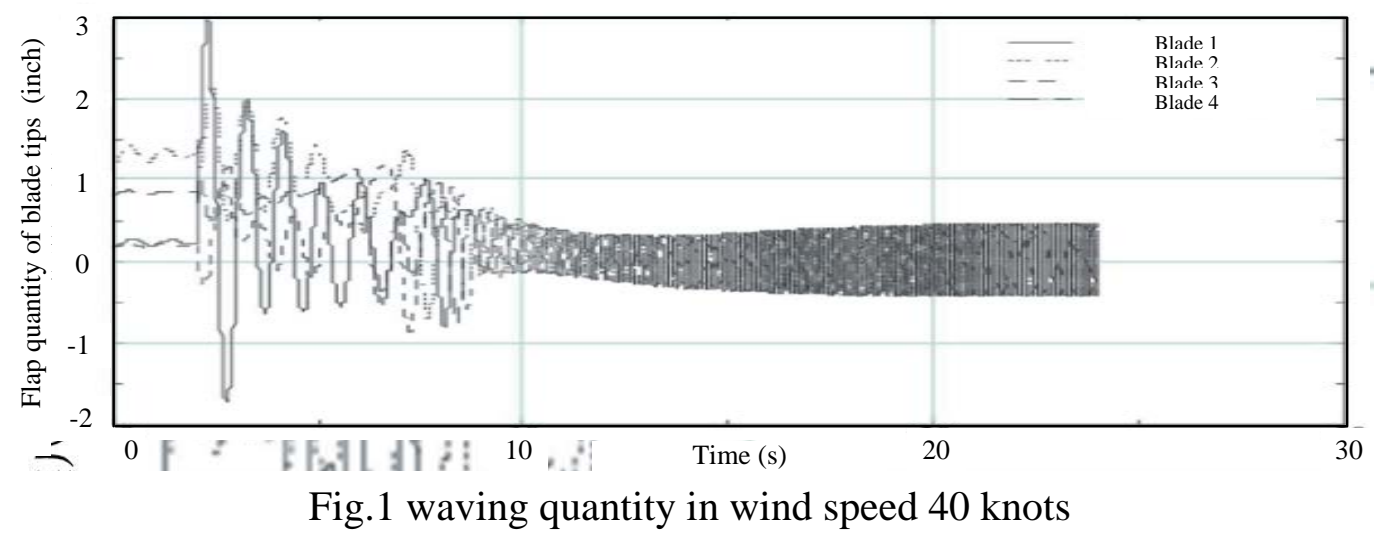

\section{Conclusion}

Results show that if suddenly suffering great gust wind, the 4 blades waving quantity would be disorder, some blades initial waving quantity are much greater, after a period of time, waving quantity is gradually stabilized.

\section{Reference}

[1] Han dong, Wang hao wen, Shang zheng. Helicopter blades raising and dropping crash dynamic response calculation. Chinese Journal of Aeronautics, 2006, 27(5): 795-798.

[2] SUN C W, The influence of shipboard flow field on helicopter operating when landing[J]. Transaction of Nanjing Aeronautical and Astronautical University, 1999 (31), 6: 614-619.

[3] WANG Shi-cun. Helicopter Aerodynamics[M], Aviation Books Association. 1985: 135-140.

[4] Gaonkar G H, Peters D A, Review of Dynamic Inflow Modeling for Rotorcraft Flight Dynamics[J]. Vertica, 1988 ,12(3): 213-242.

[5] Cheeseman I C, Bennett W E , The Effect of the Ground on a Helicopter Rotor in Forward 
Flight[R]. ARCR\&M. 1955, 3021

[6] Hong Zhang, etc. A Simulation Model of Ship Ground Effect for Rotorcraft / Ship Interaction Study[C], American Helicopter Society 51st Annual Form, 1995. 